\title{
Adaptive Integrand Decomposition
}

\section{Pierpaolo Mastrolia*}

Dipartimento di Fisica ed Astronomia, Università di Padova, and INFN, Sezione di Padova, Via Marzolo 8, 35131 Padova, Italy

pierpaolo.mastroliaepd.infn.it

\section{Tiziano Peraro}

Higgs Centre for Theoretical Physics, School of Physics and Astronomy, The University of

Edinburgh, Edinburgh EH9 3JZ, Scotland, UK

tiziano.peraro@ed.ac.uk

\section{Amedeo Primo}

Dipartimento di Fisica ed Astronomia, Università di Padova, and INFN, Sezione di Padova, Via Marzolo 8, 35131 Padova, Italy

amedeo.primolpd.infn.it

\section{William J. Torres Bobadilla}

Dipartimento di Fisica ed Astronomia, Università di Padova, and INFN, Sezione di Padova, Via Marzolo 8, 35131 Padova, Italy

william.torresepd.infn.it

We present a simplified variant of the integrand reduction algorithm for multiloop scattering amplitudes in $d=4-2 \varepsilon$ dimensions, which exploits the decomposition of the integration momenta in parallel and orthogonal subspaces, $d=d_{\|}+d_{\perp}$, where $d_{\|}$is the dimension of the space spanned by the legs of the diagrams. We discuss the advantages of a lighter polynomial division algorithm and how the orthogonality relations for Gegenbauer polynomilas can be suitably used for carrying out the integration of the irreducible monomials, which eliminates spurious integrals. Applications to one- and two-loop integrals, for arbitrary kinematics, are discussed.

Loops and Legs in Quantum Field Theory

24-29 April 2016

Leipzig, Germany

\footnotetext{
* Speaker.
} 


\section{Introduction}

The decomposition of multiloop scattering amplitudes in terms of independent functions, together with the subsequent determination of the latter, is a viable alternative to the direct integration which, for non-trivial processes, may require the calculation of a prohibitively large number of complicated Feynman integrals.

Decomposing multi-loop amplitudes in terms of independent integrals can become problematic when the number of the scales of the diagrams increases, due to the exchange or to the production of massive particles, or when a large number of external particles are scattered, or when the morphology of the contributing diagrams becomes involved. The integrand decomposition algorithm has the advantage of treating scattering amplitudes involving massive particles at the same price of amplitudes for massless scattering. The output of the reduction procedure is the partial fractioning of the original integrand, namely the determination of the remainders of the successive division between the numerator and (the partitions of the product of) the denominators. Upon inte gration, the partial fraction formula correspond to rewrite the original amplitudes as a combination of independent integrals. However, the result of the integrand decomposition represents an intermediate step towards the complete amplitude reduction. In fact, additional relations among those integrals, like integration-by-parts identities, can minimise the number of independent master integrals (MIs) which can appear in the final formulas.

The integrand decomposition algorithm $[1,2,3,4,5,6,7]$ played a key role for the automation of one-loop corrections to high-multiplicity scattering processes [8]. The extension of this approach at two-loop and beyond $[9,10,11,12]$ has been under intense investigation. The recent developments on the integrand side have been accompanied by important developments for novel derivation of the integral relations needed to identify MIs [13, 14, 15, 16], as well as by progress in the ability of computing the latter analytically $[17,18,19]$ as well as numerically $[20,21]$. This vivid research has been largely due to the deeper understanding of the properties of the integrands of Feynman graph, and of the refined algebraic and differential calculus which control them.

In these proceedings, we summarise the results of ref. [22].

\section{Parallel and orthogonal space for multiloop Feynman integrals}

In this contribution, we elaborate on a representation of dimensionally regulated Feynman integrals where, for any given diagram, the number of space-time dimensions $d(=4-2 \varepsilon)$ is split into parallel (or longitudinal) and orthogonal (or transverse) dimensions, as $d=d_{\|}+d_{\perp}$ $[23,24,25,26,27,28]$. Accordingly, the parallel space is spanned by the independent fourdimensional external momenta of the diagram, namely $d_{\|}=n-1$, where $n$ is the number of legs, whereas the transverse space is spanned by the complementary orthogonal directions. For diagrams with a number of legs $n \geq 5$, the orthogonal space embeds the $-2 \varepsilon$ regulating dimensions, $d_{\perp}=-2 \varepsilon$, while, for diagrams with $n \leq 4$, the orthogonal space is larger and it embeds, beside the regulating dimensions, also the four-dimensional complement of the parallel space, namely $d_{\perp}=(5-n)-2 \varepsilon$. In this sense, the decomposition of the space-time dimensions in parallel and orthogonal directions can be considered as adaptive, since it depends on the number of legs of the 
individual diagram.

A generic $\ell$-loop Feynman integral with $n$ external legs in a $d$-dimensional Euclidean space can be written as

$$
I_{n}^{d(\ell)}[\mathscr{N}]=\int\left(\prod_{i=1}^{\ell} \frac{d^{d} q_{i}}{\pi^{d / 2}}\right) \frac{\mathscr{N}\left(q_{i}\right)}{\prod_{j} D_{j}\left(q_{i}\right)} .
$$

In the previous equation $\mathscr{N}\left(q_{i}\right)$ is an arbitrary tensor numerator and the denominators $D_{j}\left(q_{i}\right)$ are quadratic in the loop momenta and can be written as

$$
D_{j}=l_{j}^{2}+m_{j}^{2}, \quad \text { with } \quad l_{j}^{\alpha}=\sum_{i} \alpha_{i j} q_{i}^{\alpha}+\sum_{i} \beta_{i j} p_{i}^{\alpha}
$$

where $\left\{p_{1}, \ldots, p_{n-1}\right\}$ is the set of independent external momenta and $\alpha, \beta \in\{0, \pm 1\}$.

When dealing with regularisation schemes where the external kinematics is kept in four dimensions, the $d$-dimensional loop momenta are often split into a four-dimensional part and a $(-2 \varepsilon)$ dimensional one,

$$
q_{i}^{\alpha}=q_{[4] i}^{\alpha}+\mu_{i}^{\alpha}, \quad q_{i} \cdot q_{j}=q_{[4] i} \cdot q_{[4] j}+\mu_{i j}, \quad\left(\mu_{i j}=\mu_{i} \cdot \mu_{j}\right),
$$

and the denominators read

$$
D_{i}=l_{i[4]}^{2}+\sum_{j, k} \alpha_{i j} \alpha_{i k} \mu_{j k}+m_{i}^{2}, \quad \text { with } \quad l_{i[4]}^{\alpha}=\sum_{j} \alpha_{i j} q_{i[4]}^{\alpha}+\sum_{j} \beta_{i j} p_{j}^{\alpha}
$$

Therefore both the numerator in (2.1) and the denominators become polynomials in $\ell(\ell+9) / 2$ variables, namely the $(-2 \varepsilon)$-dimensional scalar products $\mu_{i j}$ and the components of $q_{i[4]}^{\alpha}$ with respect to a four-dimensional basis of vectors $\left\{e_{i}^{\alpha}\right\}, q_{[4] i}^{\alpha}=\sum_{j=1}^{4} x_{j i} e_{j}^{\alpha}$. Thus, in $d=4-2 \varepsilon$, we can write

$$
I_{n}^{d(\ell)}[\mathscr{N}]=\Omega_{d}^{(l)} \int \prod_{i=1}^{\ell} d^{4} q_{[4] i} \int \prod_{1 \leq i \leq j \leq \ell} d \mu_{i j}\left[G\left(\mu_{i j}\right)\right]^{\frac{d-5-\ell}{2}} \frac{\mathscr{N}\left(q_{[4] i}, \mu_{i j}\right)}{\prod_{m} D_{m}\left(q_{[4] i}, \mu_{i j}\right)},
$$

where $G\left(\mu_{i j}\right)=\operatorname{det}\left[\left(\mu_{i} \cdot \mu_{j}\right)\right]$ is the Gram determinant and the prefactor $\Omega_{d}^{(\ell)}$ is the result of the angular integration over the angular directions.

For a number of external legs $n \leq 4$, the external momenta define a $d_{\|}$-dimensional subspace with $d_{\|}=n-1$. In these cases one can parametrise the integral (2.1) in such a way that the number of variables appearing in the denominators is reduced to $\ell\left(\ell+2 d_{\|}+1\right) / 2$. The numerator will instead still have a polynomial dependence over the remaining $\ell\left(4-d_{\|}\right)$variables. These can however be integrated out via a straightforward expansion of the numerator in terms of orthogonal polynomials. More in detail, if $d_{\|} \leq 3$, one can choose $4-d_{\|}$of the vectors in the basis $\left\{e_{i}^{\alpha}\right\}$ to lie into the subspace orthogonal to the external kinematics, i.e. $e_{i} \cdot p_{j}=0\left(i>d_{\|}, \forall j\right)$, and $e_{i} \cdot e_{j}=\delta_{i j}\left(i, j>d_{\|}\right)$. In this way, loop momenta in $d=d_{\|}+d_{\perp}$ read

$$
q_{i}^{\alpha}=q_{\| i}^{\alpha}+\lambda_{i}^{\alpha}
$$




$$
q_{\| i}^{\alpha}=\sum_{j=1}^{d_{\|}} x_{j i} e_{j}^{\alpha}, \quad \lambda_{i}^{\alpha}=\sum_{j=d_{\|}+1}^{4} x_{j i} e_{j}^{\alpha}+\mu_{i}^{\alpha},
$$

where $q_{\| i}$ is a vector of the $d_{\|}$-dimensional space spanned by the external momenta, and $\lambda_{i}$ belongs the $d_{\perp}$-dimensional orthogonal subspace. In this parametrisation, all denominators become independent of the transverse components of the loop momenta,

$$
D_{i}=l_{\| i}^{2}+\sum_{j, l} \alpha_{i j} \alpha_{i l} \lambda_{j l}+m_{i}^{2}, \quad l_{\| i}^{\alpha}=\sum_{j} \alpha_{i j} q_{\| i}^{\alpha}+\sum_{j} \beta_{i j} p_{j}^{\alpha}, \quad \lambda_{i j}=\sum_{l=d_{\|}+1}^{4} x_{l i} x_{l j}+\mu_{i j},
$$

and they depend on a reduced set of $\ell\left(\ell+2 d_{\|}+1\right) / 2$ variables, corresponding to the $\ell d_{\|}$components of $q_{\| i}^{\alpha}$ and the $\ell(\ell+1) / 2$ scalar products $\lambda_{i j}$. In $d=d_{\|}+d_{\perp}$, the integral (2.1) can thus be rewritten as

$$
I_{n}^{d(\ell)}[\mathscr{N}]=\Omega_{d}^{(\ell)} \int \prod_{i=1}^{\ell} d^{n-1} q_{\| i} \int d^{\frac{\ell(\ell+1)}{2}} \Lambda \int d^{\left(4-d_{\|}\right) \ell} \Theta_{\perp} \frac{\mathscr{N}\left(q_{i \|}, \Lambda, \Theta_{\perp}\right)}{\prod_{j} D_{j}\left(q_{\| i}, \Lambda\right)},
$$

where

$$
\int d^{\frac{\ell(\ell+1)}{2}} \Lambda=\int \prod_{1 \leq i \leq j} d \lambda_{i j}\left[G\left(\lambda_{i j}\right)\right]^{\frac{d_{\perp}-1-\ell}{2}}
$$

defines the integral over the norm of the transverse vectors $\lambda_{i}^{\alpha}$ and their relative orientations and $\Theta_{\perp}$ parametrises the integral over the components of $\lambda_{i}^{\alpha}$ lying in the four-dimensional space.

Remarkably, eq. (2.6) allows to express a subset of components of $q_{\| i}^{\alpha}$ and $\lambda_{i j}$ as combinations of loop denominators by solving linear relations. Therefore, one can always build differences of denominators which are linear in the loop momenta and independent of $\lambda_{i j}$, while the relation between $\lambda_{i j}$ and the denominators is always linear by definition, as it can be seen from eq. (2.8).

Since the denominators do not depend on the $\Theta_{\perp}$-components, their integration can be easily performed. In fact, the integration over $\Theta_{\perp}$ amounts to a product of factorised, univariate integrations of polynomial integrands, each of the type

$$
\int_{-1}^{1} d \cos \theta_{i j}\left(\sin \theta_{i j}\right)^{\alpha}\left(\cos \theta_{i j}\right)^{\beta}
$$

The values of $\alpha$ and $\beta$ depend on the specific expression of the numerator. Nevertheless, these integrals can be computed once and for all up to the desired rank and then re-used in every calculation, when occurring. These integrals can be evaluated by performing the Passarino-Veltman tensor reduction in the orthogonal space. Alternatively, they can be evaluated by exploiting the properties of of Gegenbauer polynomials $C_{n}^{(\alpha)}(\cos \theta)$, a particular class of orthogonal polynomials over the interval $[-1,1]$, which obey the orthogonality relation

$$
\int_{-1}^{1} d \cos \theta(\sin \theta)^{2 \alpha-1} C_{n}^{(\alpha)}(\cos \theta) C_{m}^{(\alpha)}(\cos \theta)=\delta_{m n} \frac{2^{1-2 \alpha} \pi \Gamma(n+2 \alpha)}{n !(n+\alpha) \Gamma^{2}(\alpha)} .
$$

We observe that there are special classes of multiloop integrals, associated to factorised and ladder topologies, whose denominators are independent of a certain number of transverse orientations $\lambda_{i j}$. For these cases, the Gegenbauer integration can be applied, besides to all $\Theta_{\perp}$, also to the $\lambda_{i j}$ which do not appear in the denominators. 


\section{Adaptive Integrand Decomposition}

\subsection{Integrand recurrence relation}

In the framework of the integrand reduction method $[1,3,9,10,11,29]$, the computation of dimensionally regulated $\ell$-loop integrals

$$
I_{i_{1} \ldots i_{r}}^{d(\ell)}=\int \prod_{j=1}^{\ell} \frac{d^{d} q_{j}}{\pi^{d / 2}} \frac{\mathscr{N}_{i_{1} \ldots i_{r}}\left(q_{j}\right)}{D_{i_{1}}\left(q_{j}\right) \cdots D_{i_{r}}\left(q_{j}\right)}
$$

is rephrased in terms of the reconstruction of the integrand function as a sum of integrands with irreducible numerators (or residues) and a subset of denominators $D_{i_{k}}$,

$$
\mathscr{I}_{i_{1} \ldots i_{r}}\left(q_{j}\right) \equiv \frac{\mathscr{N}_{i_{1} \ldots i_{r}}\left(q_{j}\right)}{D_{i_{1}}\left(q_{j}\right) \cdots D_{i_{r}}\left(q_{j}\right)}=\sum_{k=0}^{r} \sum_{\left\{i_{1} \cdots i_{k}\right\}} \frac{\Delta_{j_{1} \cdots j_{k}}\left(q_{j}\right)}{D_{j_{1}}\left(q_{j}\right) \cdots D_{j_{k}}\left(q_{j}\right)}
$$

For an integral with an arbitrary number $n$ of external legs, the integrand decomposition formula (3.2) can be obtained by observing that both numerator and denominators are polynomials in the components of the loop momenta with respect to some basis, which we collectively label as $\mathbf{z}=$ $\left\{z_{1}, \ldots, z_{\frac{\ell(\ell+9)}{2}}\right\}$. Thus, we can fix a monomial ordering and build a Gröebner basis $\mathscr{G}_{i_{1} \cdots i_{r}}(\mathbf{z})$ of the ideal $\mathscr{J}_{i_{1} \cdots i_{r}}$ generated by the set of denominators,

$$
\mathscr{J}_{i_{1} \cdots i_{r}} \equiv\left\{\sum_{k=1}^{r} h_{k}(\mathbf{z}) D_{i_{k}}(\mathbf{z}): h_{k}(\mathbf{z}) \in P[\mathbf{z}]\right\}
$$

being $P[\mathbf{z}]$ the ring of polynomials in $\mathbf{z}$. By performing the polynomial division of $\mathscr{N}_{i_{1} \cdots i_{r}}(\mathbf{z})$ modulo $\mathscr{G}_{i_{1} \cdots i_{r}}(\mathbf{z})$,

$$
\mathscr{N}_{i_{1} \cdots i_{r}}(\mathbf{z})=\sum_{k=1}^{r} \mathscr{N}_{i_{1} \cdots i_{k-1} i_{k+1} \cdots i_{r}}(\mathbf{z}) D_{i_{k}}(\mathbf{z})+\Delta_{i_{1} \cdots i_{r}}(\mathbf{z})
$$

we obtain the recurrence relation

$$
\mathscr{I}_{i_{1} \cdots i_{r}}=\sum_{k=1}^{r} \mathscr{I}_{i_{1} \cdots i_{k-1} i_{k+1} \cdots i_{r}}+\frac{\Delta_{i_{1} \cdots i_{r}}(\mathbf{z})}{D_{i_{1}}(\mathbf{z}) \cdots D_{i_{n}}(\mathbf{z})}
$$

whose iterative application to the integrands corresponding to subtopologies with fewer loop propagators yields to the complete decomposition (3.2).

Depending on the choice of variables $\mathbf{z}$ and the monomial order, the picture presented in this section can significantly simplify. A particular convenient choice of variables turns out to be the one presented in eq. (2.6). Indeed, as already observed, we can always express a subset of the components of $q_{\| i}^{\alpha}$ and $\lambda_{i j}$ as a combination of denominators by solving linear relations. This set of relations is in turn equivalent to the definition of the denominators themselves. This implies that if we choose the lexicographic monomial order with $\lambda_{i j} \prec x_{k l}$ for $k \leq d_{\|}$, the polynomials in the Gröbner bases are linear in the $\lambda_{i j}$ and the reducible components of $q_{\| i}^{\alpha}$. The polynomial division can thus equivalently be performed by applying the aforementioned set of linear relations without explicitly computing the corresponding Gröbner basis. 


\subsection{Divide, integrate and divide}

When dealing with an integral with $n \leq 4$ external legs, we can use the $d=d_{\|}+d_{\perp}$ parametrisation which removes the dependence of the denominators on the transverse components of the loop momenta. Thus, if we indicate with $\mathbf{z}$ the full set of $\ell(\ell+9) / 2$ variables

$$
\mathbf{z}=\left\{\mathbf{x}_{\| i}, \mathbf{x}_{\perp i}, \lambda_{i j}\right\}, \quad i, j=1, \ldots \ell,
$$

where $\mathbf{x}_{\| i}\left(\mathbf{x}_{\perp i}\right)$ are the components of the loop momenta parallel(orthogonal) to the external kinematics, the denominators are reduced to polynomials in the subset of variables

$$
\tau=\left\{\mathbf{x}_{\|}, \lambda_{i j}\right\}, \quad \tau \subset \mathbf{z},
$$

so that the general $r$ denominators integrand has the form

$$
\mathscr{I}_{i_{1} \ldots i_{r}}\left(\tau, \mathbf{x}_{\perp}\right) \equiv \frac{\mathscr{N}_{i_{1} \ldots i_{r}}\left(\tau, \mathbf{x}_{\perp}\right)}{D_{i_{1}}(\tau) \cdots D_{i_{r}}(\tau)} .
$$

This observation suggests an adaptive version of the integrand decomposition algorithm, where the polynomial division is simplified by working on the reduced set of variables $\tau$ and the expansion of the residues in terms of Gegenbauer polynomials allows the systematic identification of spurious terms. The algorithm is organised in three steps:

1. Divide: we adopt lexicographic ordering $\lambda_{i j} \prec \mathbf{x}_{\|}$for the $\tau$ variables and we divide the numerator $\mathscr{N}_{i_{1} \ldots i_{r}}\left(\tau, \mathbf{x}_{\perp}\right)$ modulo the Gröebner basis $\mathscr{G}_{i_{1} \cdots i_{r}}(\tau)$ of the ideal $\mathscr{J}_{i_{1} \cdots i_{r}}(\tau)$ generated by the denominators,

$$
\mathscr{N}_{i_{1} \ldots i_{r}}\left(\tau, \mathbf{x}_{\perp}\right)=\sum_{k=1}^{r} \mathscr{N}_{i_{1} \ldots i_{k-1} i_{k+1} \ldots i_{r}}\left(\tau, \mathbf{x}_{\perp}\right) D_{i_{k}}(\tau)+\Delta_{i_{1} \ldots i_{r}}\left(\mathbf{x}_{\|}, \mathbf{x}_{\perp}\right) .
$$

As a consequence of the specific monomial ordering, the residue $\Delta_{i_{1} \ldots i_{r}}$ can depend on the transverse components $\mathbf{x}_{\perp i}$, which are left untouched by the polynomial division, as well as on $\mathbf{x}_{\| i}$ but not on $\lambda_{i j}$ that are expressed in terms of denominators and irreducible physical scalar products. Conversely, the quotient, from which the numerators corresponding to the subdiagrams to be further divided are obtained, still depends on the full set of loop variables. As we explained at the end of sec. 3.1, the Gröbner basis does not need to be explicitly computed, since, with the choice of variables and the ordering described here, the division is equivalent to applying the set of linear relations described above.

2. Integrate: by writing the contribution of the residue $\Delta_{i_{1} \ldots i_{r}}$ to the integral in the $d=d_{\|}+d_{\perp}$ parametrisation, we can integrate over transverse directions through the expansion of $\Delta_{i_{1} \ldots i_{r}}$ in terms of Gegenbauer polynomials, which sets to zero spurious terms and reduce all nonvanishing contributions to monomials in $\lambda_{i j}$. It should be noted that, due to the angular prefactors produced by the integration of the transverse directions, the integrated residue

$$
\Delta_{i_{1} \ldots i_{r}}^{\mathrm{int}}(\tau)=\int d^{\left(4-d_{\|}\right) \ell} \Theta_{\perp} \Delta_{i_{1} \ldots i_{r}}\left(\tau, \Theta_{\perp}\right)
$$

is, in general, a polynomial in $\tau$ whose coefficients depend explicitly on the space-time dimension $d$. The full set of $\Delta_{i_{1} \ldots i_{r}}^{\mathrm{int}}(\tau)$, obtained by iterating on each subdiagram numerator the polynomial division and the integration over the transverse space, provides already a spurious term-free representation of the integrand (3.2). 
3. Divide: however, since $\Delta_{i_{1} \ldots i_{r}}^{\mathrm{int}}(\tau)$ depends on the same variables as the denominators $D_{i_{k}}(\tau)$, we can perform a further division modulo the Gröebner basis $\mathscr{G}_{i_{1} \cdots i_{r}}(\tau)$ and get

$$
\Delta_{i_{1} \ldots i_{r}}^{\mathrm{int}}(\tau)=\sum_{k=1}^{r} \mathscr{N}_{i_{1} \ldots i_{k-1} i_{k+1} \ldots i_{r}}^{\mathrm{int}}(\tau) D_{i_{k}}(\tau)+\Delta_{i_{1} \ldots i_{r}}^{\prime}\left(\mathbf{x}_{\|}\right)
$$

where, due to the choice of lexicographic ordering, the new residue $\Delta_{i_{1} \ldots i_{r}}^{\prime}\left(\mathbf{x}_{\|}\right)$can only depend on $\mathbf{x}_{\|}$. Therefore, this additional polynomial division allows us to obtain an integrand decomposition formula (3.2), where all irreducible numerators are function of the components of the loop momenta parallel to the external kinematics. As in the previous case, the division modulo Gröbner can equivalently be implemented via a set of linear relations.

The interpretation of the monomials appearing in the residues $\Delta_{i_{1} \cdots i_{r}}^{\prime}\left(\mathbf{x}_{\|}\right)$in terms of a basis of tensor integrals can be additionally simplified by making use of the Gram determinant $G\left[\lambda_{i j}\right]$ (or $G\left[\mu_{i j}\right]$ for cases with more than four external legs, where $\mathbf{x}_{\|} \equiv \mathbf{x}$ ). In fact, as it can be easily understood from (2.5) and (2.10a), $G\left[\mu_{i j}\right]$ and $G\left[\lambda_{i j}\right]$ can be interpreted as operators that, when acting on an arbitrary numerator of a $d$-dimensional integral, produce a dimensional shift $d \rightarrow d+2$. Therefore, Gram determinants can be used in order to trade some of the $d$-dimensional tensor integrals originating from the residues with lower rank integrals in higher dimensions.

\subsection{Integrate and divide}

In the three-step algorithm divide-integrate-divide, outlined in the previous section, the integration over the transverse angles is performed after the integrand reduction, namely after determining the residues. This first option follows the standard integrand reduction procedure, where the spurious monomials are present in the decomposed integrand, although they do not contribute to the integrated amplitude. Alternatively, if the dependence of the numerators on the loop momenta is known, then the integration over the orthogonal angles can be carried out before the reduction. Within this second option, which we can refer to as integrate-divide, after eliminating the orthogonal angles from the integrands, the residues resulting from the polynomial divisions contain only non-spurious monomials. In order to integrate before the reduction, the dependence of the numerator on the loop momenta should be either known analytically or reconstructed semi-analytically $[30,31]$. Such situation may indeed occur when the integrands to be reduced are built by automatic generators or they emerge as quotients of the subsequent divisions.

\section{Applications}

We summarise the results obtained from the application of the adaptive integrand decomposition (AID) at one loop in Table 1. In the first column, $\tau$ labels the variables the denominators depend on. $\Delta_{i_{1} \cdots i_{n}}$ indicates the residue obtained after the polynomial division of an arbitrary $n$ rank numerator and $\Delta_{i_{1} \cdots i_{n}}^{\text {int }}$ the result of its integral over transverse directions. $\Delta_{i_{1} \cdots i_{n}}^{\prime}$ corresponds to the minimal residue obtained from a further division of $\Delta_{i_{1} \cdots i_{n}}^{\mathrm{int}}$. In the figures, wavy lines indicate massless particles, whereas solid ones stands for arbitrary masses. As an exceptional property of one-loop integrands, we find that by working with $\tau$ variables, all unitarity cuts are reduced to zero-dimensional systems. Moreover, we show that the last step of the algorithm, i.e. the further 


\begin{tabular}{|c|c|c|c|c|}
\hline $\mathscr{I}_{i_{1} \cdots i_{n}}$ & $\tau$ & $\Delta_{i_{1} \cdots i_{n}}$ & $\Delta_{i_{1} \cdots i_{n}}^{\mathrm{int}}$ & $\Delta_{i_{1} \cdots i_{n}}^{\prime}$ \\
\hline $\mathscr{I}_{i_{1} i_{2} i_{3} i_{4} i_{5}}$ & $\left\{x_{1}, x_{2}, x_{3}, x_{4}, \mu^{2}\right\}$ & $\begin{array}{c}1 \\
\{1\}\end{array}$ & - & $\begin{array}{l}- \\
-\end{array}$ \\
\hline $\mathscr{I}_{i_{1} i_{2} i_{3} i_{4}}$ & $\left\{x_{1}, x_{2}, x_{3}, \lambda^{2}\right\}$ & $\begin{array}{c}5 \\
\left\{1, x_{4}, x_{4}^{2}, x_{4}^{3}, x_{4}^{4}\right\} \\
\end{array}$ & $\begin{array}{c}3 \\
\left\{1, \lambda^{2}, \lambda^{4}\right\}\end{array}$ & $\begin{array}{c}1 \\
\{1\}\end{array}$ \\
\hline $\mathscr{I}_{i_{1} i_{2} i_{3}}$ & $\left\{x_{1}, x_{2}, \lambda^{2}\right\}$ & $\begin{array}{c}10 \\
\left\{1, x_{3}, x_{4}, x_{3}^{2}, x_{3} x_{4}, x_{4}^{2}, x_{3}^{3}, x_{3}^{2} x_{4}, x_{3} x_{4}^{2}, x_{4}^{3}\right\}\end{array}$ & $\begin{array}{c}2 \\
\left\{1, \lambda^{2}\right\}\end{array}$ & $\begin{array}{c}1 \\
\{1\} \\
\end{array}$ \\
\hline $\mathscr{I}_{i_{1} i_{2}}$ & $\left\{x_{1}, \lambda^{2}\right\}$ & $\begin{array}{c}10 \\
\left\{1, x_{2}, x_{3}, x_{4}, x_{2}^{2}, x_{2} x_{3}, x_{2} x_{4}, x_{3}^{2}, x_{3} x_{4}, x_{4}^{2}\right\}\end{array}$ & $\begin{array}{c}2 \\
\left\{1, \lambda^{2}\right\}\end{array}$ & $\begin{array}{c}1 \\
\{1\}\end{array}$ \\
\hline $\mathscr{I}_{i_{1} i_{2}}$ & $\left\{x_{1}, x_{2}, \lambda^{2}\right\}$ & $\begin{array}{c}10 \\
\left\{1, x_{1}, x_{3}, x_{4}, x_{1}^{2}, x_{1} x_{3}, x_{1} x_{4}, x_{3}^{2}, x_{3} x_{4}, x_{4}^{2}\right\}\end{array}$ & $\begin{array}{c}4 \\
\left\{1, x_{1}, x_{1}^{2}, \lambda^{2}\right\} \\
\end{array}$ & $\begin{array}{c}3 \\
\left\{1, x_{1}, x_{1}^{2}\right\}\end{array}$ \\
\hline $\mathscr{I}_{i_{1}}$ & $\left\{\lambda^{2}\right\}$ & $\begin{array}{c}5 \\
\left\{1, x_{1}, x_{2}, x_{3}, x_{4}\right\}\end{array}$ & $\begin{array}{c}1 \\
\{1\}\end{array}$ & - \\
\hline
\end{tabular}

Table 1: Residue parametrisation for irreducible one-loop topologies.

polynomial division after angular integration over the transverse space, provides an implementation of the dimensional recurrence relations at the integrand level.

Beside revisiting the one-loop, we applied the AID in order to determine the universal parametrisation of the residues appearing in the integrand decomposition (3.2) of the three eight-point topologies shown in fig. 1a-1c. The results obtained are valid for arbitrary (internal and external) kinematic configuration. For the complete results of the two-loop decomposition, we refer the reader to the ref. [22].

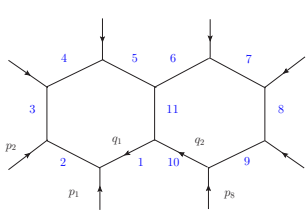

(a) $\mathscr{I}_{1234567891011}^{\mathrm{P}}$

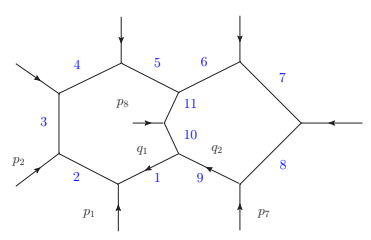

(b) $\mathscr{I}_{1234567891011}^{\mathrm{NP}}$

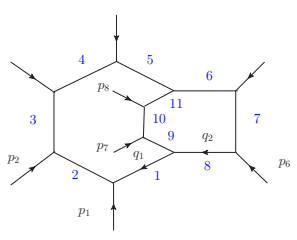

(c) $\mathscr{I}_{1234567891011}^{\mathrm{NP}}$

Figure 1: Maximum-cut topologies

Furthermore, we applied the AID to the leading color contribution to the two-loop all-plus fivegluon amplitude [32, 33, 34, 35, 36, 37, 38], which, after the first step of the division algorithm, admits a decomposition of the form

$$
\begin{aligned}
& A^{(2)}\left(1^{+}, 2^{+}, 3^{+}, 4^{+}, 5^{+}\right)=\int \frac{d^{d} q_{1}}{\pi^{d / 2}} \frac{d^{d} q_{2}}{\pi^{d / 2}}\left\{\frac{\Delta\left(\begin{array}{c}
5 \\
{ }_{4}-L_{-2}^{1}
\end{array}\right)}{D_{1} D_{2} D_{3} D_{4} D_{5} D_{6} D_{7} D_{8}}+\frac{\Delta\left(\begin{array}{l}
5 \\
{ }_{4} L_{-2}^{1}
\end{array}\right)}{D_{1} D_{2} D_{3} D_{4} D_{5} D_{6} D_{7}}\right.
\end{aligned}
$$

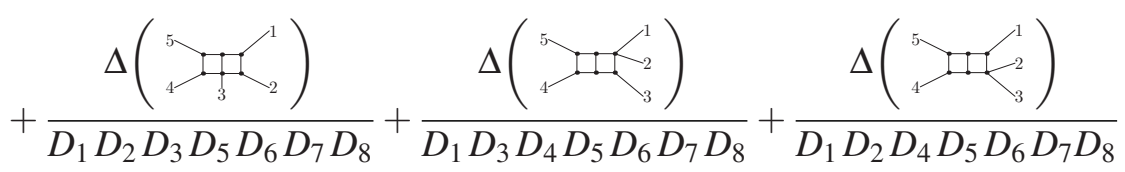




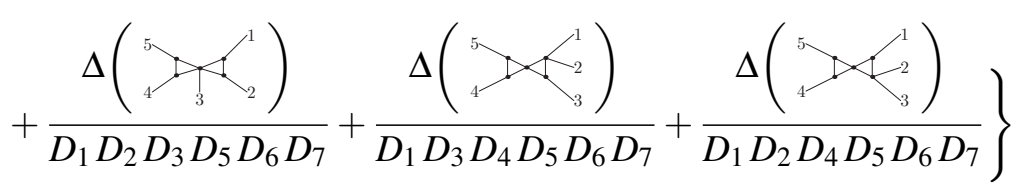

$$
\begin{aligned}
& + \text { cycl. perm. }
\end{aligned}
$$

The residue have been obtained from numerators constructed through Feynman diagrams in Feynman gauge, including both gluon and ghost loop contributions. The relevant Feynman graphs, a selection of which is shown in fig. 2, have been generated by using FEYNARTS [39] and FEYNCALC [40, 41]. The expression of the residues have been numerically checked against the results of [32]. The integration of the transverse directions of both four-point and factorised topologies and the further division of the integrated residues may lead to a new representation of the amplitude, whose discussion is, nevertheless, beyond the scope of this report.

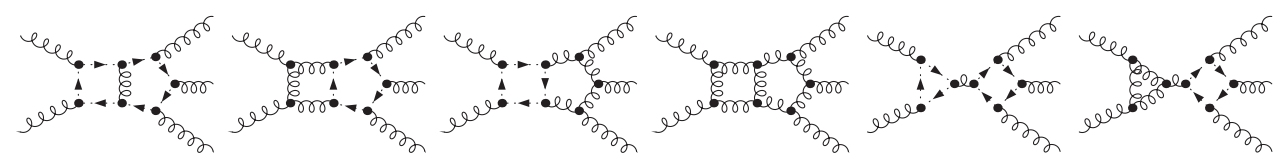

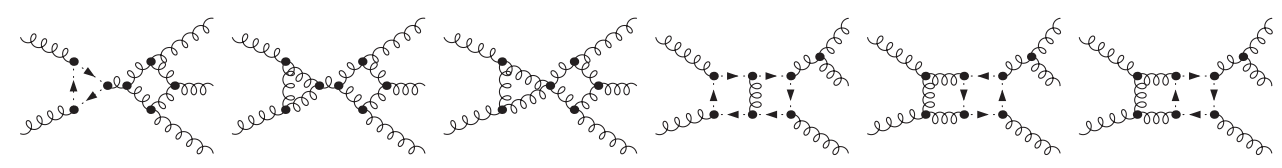

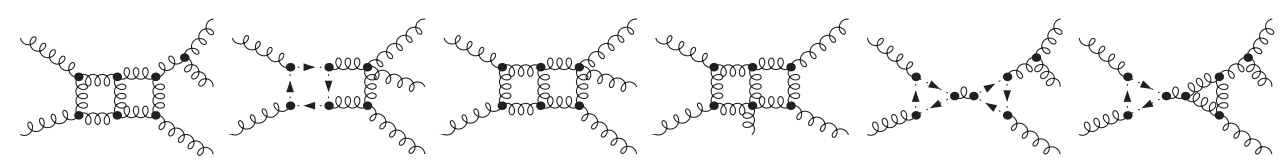

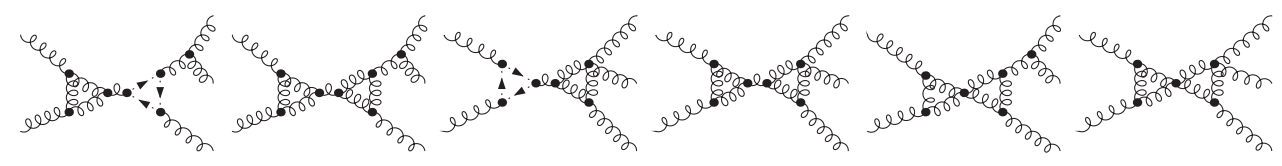

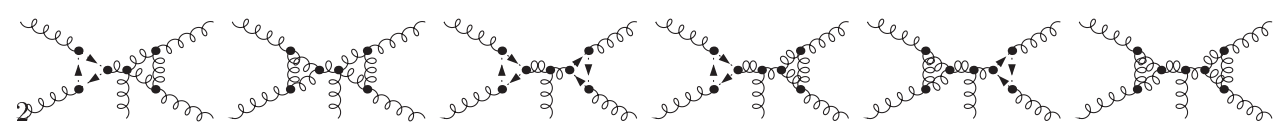

Figure 2: Selection of Feynman diagrams contributing to the five-gluons amplitude. Curly lines represent gluons and dashed ones indicate ghosts.

\section{Conclusions}

Owing to the representation of Feynman integrals in parallel and orthogonal space, numerators and denominators of integrands appear to depend on different sets of integration variables. By exploiting the different origin and role of these variables, we engineered a novel variant of the integrand decomposition algorithm, defined as adaptive integrand decomposition (AID), where the multivariate polynomial division is simplified and the integration over transverse space variables 
can be efficiently carried out by means of Gegenbauer polynomials. Orthogonality relations for Gegenbauer polynomials detect and annihilate the so called spurious integrals at any loop order, and can be used any time that a certain subset of integration variables do not appear in the denominators, as it happens also in the case of factorised diagrams and ladder topologies.

As a result of the AID, each amplitude is written in terms of a set of integrals which, beside the scalar ones, contains tensor integrals with irreducible scalar products depending on the parallel directions and on the lengths of the transverse vectors only. In addition, we have shown that the integration over the transverse directions leads to integrals which can be subject to additional polynomial divisions, which in some cases correspond to dimension-shifting recurrence relations implemented at the integrand level.

We revisited the one-loop integrand decomposition, showing that it is completely determined by the maximum-cut theorem in different dimensions. Furthermore, we considered the complete reduction of two-loop planar and non-planar integrals for arbitrary kinematics, classifying the corresponding residues and identifying the independent integrals contributing to eight-particle scattering amplitudes. The proposed algorithm can be simply extended to higher loops.

In view of the development of automated tools for the evaluation of multi-loop amplitudes, the AID can be used as an intermediate reduction phase to achieve an expression in terms of an independent set of integrals. The latter can be further simplified by means of relations arising from additional symmetries that can occur, such as integration-by-parts identities and color-kinematics duality.

\section{References}

[1] G. Ossola, C. G. Papadopoulos, and R. Pittau Nucl. Phys. B763 (2007) 147-169, [hep-ph/0609007].

[2] G. Ossola, C. G. Papadopoulos, and R. Pittau JHEP 07 (2007) 085, [0 704 . 1271 ].

[3] R. K. Ellis, W. T. Giele, and Z. Kunszt JHEP 03 (2008) 003, [0 708 . 2398].

[4] R. K. Ellis, W. T. Giele, Z. Kunszt, and K. Melnikov Nucl. Phys. B822 (2009) 270-282, [0806.3467].

[5] G. Ossola, C. G. Papadopoulos, and R. Pittau JHEP 05 (2008) 004, [0 802 . 1876 ].

[6] P. Mastrolia, G. Ossola, C. G. Papadopoulos, and R. Pittau JHEP 06 (2008) 030, [0 803.3964 ].

[7] P. Mastrolia, E. Mirabella, and T. Peraro JHEP 06 (2012) 095, [1203 . 02 91]. [Erratum: JHEP11,128(2012)].

[8] G. Ossola These proceedings.

[9] P. Mastrolia and G. Ossola JHEP 11 (2011) 014, [1107. 6041$].$

[10] S. Badger, H. Frellesvig, and Y. Zhang JHEP 04 (2012) 055, [1202 . 2019].

[11] Y. Zhang JHEP 09 (2012) 042, [1205. 5707].

[12] P. Mastrolia, E. Mirabella, G. Ossola, and T. Peraro Phys. Lett. B718 (2012) 173-177, [1205 . 7087 ].

[13] H. Ita 1510.05626.

[14] K. J. Larsen and Y. Zhang Phys. Rev. D93 (2016), no. 4 041701, [1511. 01071$].$ 
[15] A. von Manteuffel and R. M. Schabinger Phys. Lett. B744 (2015) 101-104, [1 406.4513 ].

[16] P. Kant Comput. Phys. Commun. 185 (2014) 1473-1476, [1309. 7287].

[17] J. M. Henn Phys. Rev. Lett. 110 (2013) 251601, [1304.1806].

[18] M. Argeri, S. Di Vita, P. Mastrolia, E. Mirabella, J. Schlenk, U. Schubert, and L. Tancredi JHEP 03 (2014) 082, [1401.2979].

[19] C. G. Papadopoulos JHEP 07 (2014) 088, [1401. 6057].

[20] S. Borowka, G. Heinrich, S. P. Jones, M. Kerner, J. Schlenk, and T. Zirke Comput. Phys. Commun. 196 (2015) 470-491, [1502.06595].

[21] A. V. Smirnov Comput. Phys. Commun. 204 (2016) 189-199, [1511. 03614$].$

[22] P. Mastrolia, T. Peraro, and A. Primo 1605.03157.

[23] J. C. Collins, Renormalization: an introduction to renormalization, the renormalization group, and the operator-product expansion. Cambridge monographs on mathematical physics. Cambridge Univ. Press, Cambridge, 1984.

[24] D. Kreimer Z. Phys. C54 (1992) 667-672.

[25] D. Kreimer Phys. Lett. B292 (1992) 341-347.

[26] A. Czarnecki, U. Kilian, and D. Kreimer Nucl. Phys. B433 (1995) 259-275, [hep-ph/ 9405423 ].

[27] A. Frink, U. Kilian, and D. Kreimer Nucl. Phys. B488 (1997) 426-440, [hep-ph/961 0285 ].

[28] D. Kreimer Nucl. Instrum. Meth. A389 (1997) 323-326.

[29] P. Mastrolia, E. Mirabella, G. Ossola, and T. Peraro Phys. Lett. B727 (2013) 532-535, [1307. 5832 ].

[30] G. Heinrich, G. Ossola, T. Reiter, and F. Tramontano JHEP 10 (2010) 105, [1 008.2441$].$

[31] V. Hirschi and T. Peraro 1604.01363.

[32] S. Badger, H. Frellesvig, and Y. Zhang JHEP 12 (2013) 045, [1310 . 1051$].$

[33] S. Badger, G. Mogull, A. Ochirov, and D. O’Connell JHEP 10 (2015) 064, [1507. 08797 ].

[34] C. G. Papadopoulos, D. Tommasini, and C. Wever JHEP 04 (2016) 078, [1 511.09404 ].

[35] T. Gehrmann, J. M. Henn, and N. A. Lo Presti Phys. Rev. Lett. 116 (2016), no. 6062001 , [1511.05409]. [Erratum: Phys. Rev. Lett.116,no.18,189903(2016)].

[36] D. C. Dunbar and W. B. Perkins Phys. Rev. D93 (2016), no. 8 085029, [1603. 07514 ].

[37] D. C. Dunbar, G. R. Jehu, and W. B. Perkins Phys. Rev. D93 (2016), no. 12 125006, [1604. 06631 ].

[38] S. Badger, G. Mogull, and T. Peraro 1606.02244.

[39] T. Hahn Comput. Phys. Commun. 140 (2001) 418-431, [hep-ph/ 0012260 ].

[40] R. Mertig, M. Bohm, and A. Denner Comput. Phys. Commun. 64 (1991) 345-359.

[41] V. Shtabovenko, R. Mertig, and F. Orellana 1601.01167. 